\title{
Mechanisms of non-contact anterior cruciate ligament injury as determined by bone contusion location and severity
}

\author{
Lanyu Qiu", Bo Sheng", Jia Li, Zhibo Xiao, Mao Yuan, Haitao Yang, Fajin Lv, Furong Lv \\ Department of Radiology, The First Affiliated Hospital of Chongqing Medical University, Chongqing, China
}

\#These authors contributed equally to this work.

Correspondence to: Furong Lv. Department of Radiology, The First Affiliated Hospital of Chongqing Medical University, No. 1 Youyi Road, Yuanjiagang, Yuzhong District, Chongqing 400016, China. Email: lfr918@sina.com.

\begin{abstract}
Background: The location and severity of tibiofemoral bone contusions in magnetic resonance imaging scans in patients with acute non-contact anterior cruciate ligament injuries can reflect the primary mechanisms of anterior cruciate ligament injuries. There has been limited investigation to subdividing the bone contusion model in the medial and lateral directions of the tibial plateau and the femoral condyle.

Methods: A retrospective review of 93 consecutive magnetic resonance imaging examinations of patients with acute non-contact anterior cruciate ligament injuries was conducted to identify bone contusions of the knee. The locations and the severity of the bone contusions were determined using magnetic resonance imaging scans for each anatomic site, including the lateral femoral condyle, the lateral tibial plateau, the medial femoral condyle, and the medial tibial plateau. The bone contusions in the lateral-medial and anteriorposterior directions of four anatomical sites were subdivided into six compartments. The severity of the bone contusions was graded on a scale of $1-4$. The location and the severity of bone contusions were accessed in the sagittal and coronal planes on the femoral and tibial sides of the knee using the radiology information system.

Results: The prevalence of bone contusions on the magnetic resonance imaging scans was as follows: $78.49 \%$ on the lateral femoral condyle, $88.17 \%$ on the lateral tibial plateau, $49.46 \%$ on the medial femoral condyle, and $69.89 \%$ on the medial tibial plateau. The most common and severe compartments of the lateral femoral condyle, the lateral tibial plateau, the medial femoral condyle, and the medial tibial plateau were the centrallateral (CL), the posterior-medial (PM), the CL, and the posterior-lateral (PL) compartments, respectively.

Conclusions: The location patterns and severity of bone contusions in patients indicated that internal tibial rotation, valgus, and the anterior and lateral translation of the tibia were the primary mechanisms of non-contact anterior cruciate ligament injury.
\end{abstract}

Keywords: Bone contusion; anterior cruciate ligament; kissing contusion; mechanisms of injury; noncontact injury

Submitted Oct 28, 2020. Accepted for publication Apr 09, 2021.

doi: $10.21037 /$ qims-20-1212

View this article at: http://dx.doi.org/10.21037/qims-20-1212

\section{Introduction}

Anterior cruciate ligament injury is one of the most common sports injuries. Prevention programs have focused on avoiding non-contact anterior cruciate ligament injuries, which account for almost $3 / 4$ of all anterior cruciate ligament injuries $(1,2)$. The mechanisms of an anterior cruciate ligament injury are complex and involve a multiplanar (rather than a single) loading pattern (3-6). The most common noncontact anterior cruciate ligament injury mechanism is knee valgus combined with the knee rotation $(4,5)$; however, the types of rotation that cause anterior cruciate ligament injury are not completely clear. Some video analysis studies have observed both internal and external rotations during anterior cruciate ligament injuries $(5,7,8)$, while other studies have 
shown that external rotation does not increase anterior cruciate ligament load (9). Due to the conflicting theories and limited data on the precise motions responsible for noncontact anterior cruciate ligament injuries, there is currently a lack of information regarding the rotations that cause an anterior cruciate ligament injury. Understanding the rotation patterns leading to anterior cruciate ligament rupture is particularly important and could help identify individuals at high risk of injury.

Bone contusions are believed to result from trabecular damage due to impact between the articular surfaces at the time of injury $(10,11)$. Magnetic resonance imaging has moderate to high sensitivity in identifying bone contusions (12), which are commonly observed in patients with non-contact rather than contact injuries (6). Notably, the non-contact mechanisms appear to cause more severe and more frequent bone contusions in both the medial and lateral sites. It has been suggested that bone contusions observed on magnetic resonance imaging scans in the tibia and femur are secondary signs for detecting other associated abnormalities and the mechanisms of injury (13-16). The degree of bone contusions may relate to the amount of energy imparted on the knee during the injury $(6,17)$. Comprehensive investigations into the precise location and severity of the bone contusions may increase understanding of anterior cruciate ligament injury mechanisms and assist in the development of more appropriate and effective preventative measures.

Tibiofemoral bone contusions are believed to result from joint impact at the time of anterior cruciate ligament rupture. Previous studies have used the location, volume, intensity, depth, and frequency at which a bone contusion occurs (as observed in the magnetic resonance imaging scans of individuals with anterior cruciate ligament injury) to elucidate the injury mechanisms. Kissing contusions are defined as relative bone contusion patterns in the tibia and femur and have been the focus of research into anterior cruciate ligament injury mechanisms (18). A recent study on the location of bone contusions in the anterior-posterior direction found that the anterior translation of the tibia relative to the femur occurs during anterior cruciate ligament injury (17). In addition, the lateral femoral condyle and lateral tibial plateau were found to be more common in patients with non-contact anterior cruciate ligament injuries than those with contact injuries (6). Bone contusions are often present in the posterior side of the tibial plateau and the femoral condyle's central site, indicating that the mechanisms are more consistent with the tibia's anterior translation femur and internal tibial rotation (6). Previous analyses have provided valuable information on the mechanisms of anterior cruciate ligament injury-based bone contusion patterns. However, only bone contusion locations in the anterior-posterior direction have been described, and locations in each anatomic site's medial-lateral direction have not yet been investigated.

In our clinical practice, we have found differences in the bone contusion locations in the medial-lateral direction of the tibial plateau in patients with non-contact anterior cruciate ligament injuries. Based on these data, we aimed to explore differences in the medial and lateral distribution of posterior tibial plateau bone contusions and determine if anterior cruciate ligament injury mechanisms can be inferred from these differences. The mechanisms of anterior cruciate ligament injury remain controversial, particularly in so far as these mechanisms concern rotation. By subdividing the bone contusion model in the medial and lateral directions of the tibial plateau and the femoral condyle, new information about potential anterior cruciate ligament injury mechanisms with more degrees of freedom (DOF) may be gathered. This study sought to investigate non-contact anterior cruciate ligament injury mechanisms by analyzing the location and severity of bone contusions in the lateral-medial and anterior-posterior directions of the distal femur and proximal tibia.

\section{Methods}

\section{Patient selection}

A retrospective analysis was performed of 686 consecutive patients who had been clinically diagnosed with anterior cruciate ligament injuries and had undergone primary anterior cruciate ligament reconstruction between January 2014 and June 2020. Patients' medical and surgical records, and magnetic resonance imaging scans were reviewed. Patients were eligible to participate in this study if they had primary anterior cruciate ligament reconstructions relative to lesions resulting from a non-contact mechanism of injury and had undergone an magnetic resonance imaging examination no longer than 1 month after the initial injury. In this study, a non-contact injury was defined as an anterior cruciate ligament injury that did not involve any external forces other than the ground reaction force (19). As part of the selection criteria, bone contusions had to be visible in the femur or tibia. A bone contusion was defined as edema in the trabecular bone marrow with an area of high-signal 


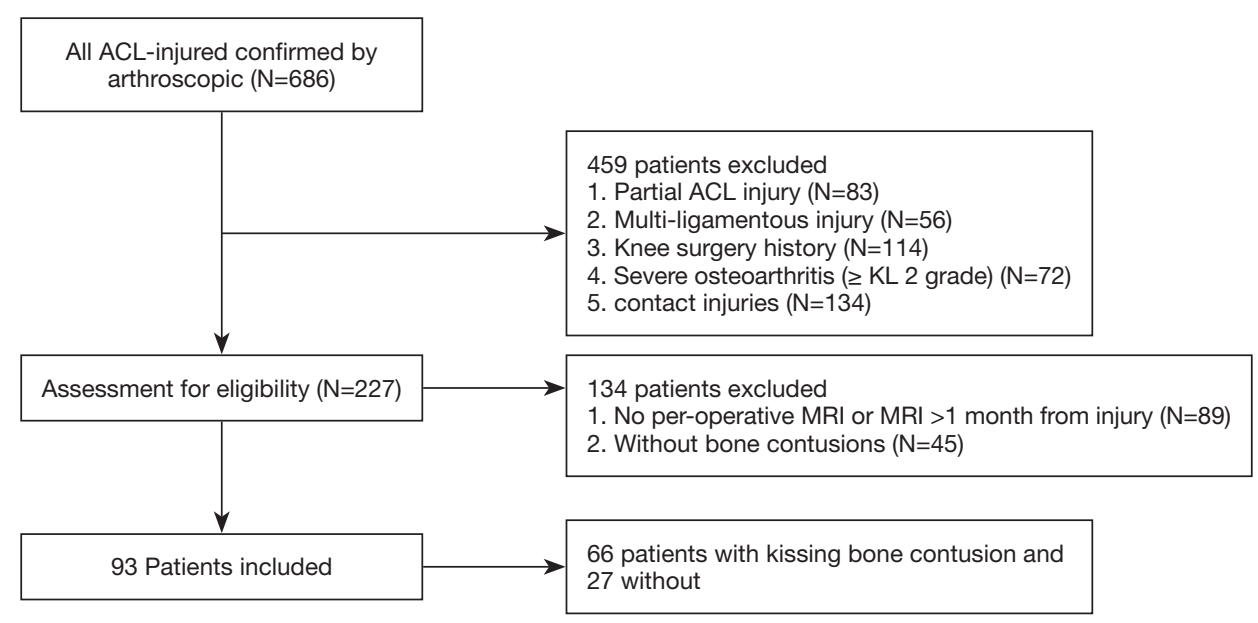

Figure 1 A flowchart for the selection of patients. ACL, anterior cruciate ligament; KL, Kellgren-Lawrence; MRI, magnetic resonance imaging.

intensity on T2- or proton density-weighted fat-suppressed images and low signal intensity on T1-weighted images. The exclusion criteria were as follows: (I) a partial anterior cruciate ligament injury; (II) a history of knee surgery on the affected side; (III) a lack of available preoperative magnetic resonance imaging scans for accurate image evaluation; (IV) the presence of severe osteoarthritis [ $\geq$ Kellgren-Lawrence (KL) 2 grade], or inflammatory arthritis; and/or (V) a combined posterior cruciate ligament injury. The location and severity of bone contusions of the tibia and femur were recorded. A flow chart showing the selection of patients is presented in Figure 1. Our institution's Committee approved this study for Human Research.

\section{Magnetic resonance imaging protocol}

The magnetic resonance imaging scans were performed on 1.5-T magnetic resonance imaging equipment (Magnetom Essenza; Siemens Healthcare) using an extremity matrix knee coil (Tim coil). A standard magnetic resonance imaging protocol was used, which included sagittal and coronal T1 turbo spin-echo [repetition time (TR), $306 \mathrm{~ms}$; echo time (TE), $12 \mathrm{~ms}$; the field of view (FOV), $16 \mathrm{~cm}$; matrix size, $320 \times 320$; slice thickness, $4 \mathrm{~mm}$ ], sagittal T2 turbo spin-echo (TR, 3,220 ms; TE, $99 \mathrm{~ms}$; FOV, $16 \mathrm{~cm}$; matrix size, $320 \times 320$; slice thickness, $4 \mathrm{~mm}$ ), sagittal and coronal intermediate-weighted turbo spin-echo with fat saturation (TR, 2,800 ms; TE, $38 \mathrm{~ms}$; FOV, $16 \mathrm{~cm}$; matrix size, $256 \times 256$; slice thickness, $4 \mathrm{~mm}$ ), and axial intermediate-weighted turbo spin-echo with fat saturation
(TR, 2,800 ms; TE, $51 \mathrm{~ms}$; FOV, $16 \mathrm{~cm}$; matrix size, $232 \times 256$; slice thickness, $4 \mathrm{~mm}$ ).

\section{Determining the location and severity of bone contusions}

The patterns of the tibiofemoral bone contusions were classified and analyzed. Anatomical sites of bone contusions included the lateral femoral condyle, the lateral tibial plateau, the medial femoral condyle, and the medial tibial plateau. The bone contusions of each anatomical site were divided into the following six compartments: (I) the anterior-medial (AM); (II) the central-medial (CM), (III) the posterior-medial (PM), (IV) the anterior-lateral (AL), (V) the central-lateral (CL), (VI) the posterior-lateral (PL).

Imaging sequences were used to determine the location of bone contusions in the lateral-medial and anteriorposterior directions. The location of bone contusions in the lateral-medial direction was first determined using the coronal sequence (see Figure $2 A$ ), and the location of bone contusions in the anterior-posterior direction was then determined using the sagittal sequence (see Figure 2B). The lines dividing the sub-compartments were perpendicular to the respective subchondral bone. Additionally, the severity of bone contusions was identified and divided into four grades based on the depth of bone contusion on the fat-suppressed magnetic resonance imagings (6). The four grades were as follows: superficial (Grade 1), shallow (Grade 2), deep (Grade 3), and generalized (Grade 4). The classification of bone contusion is shown in Table 1. All magnetic resonance imaging images were independently 

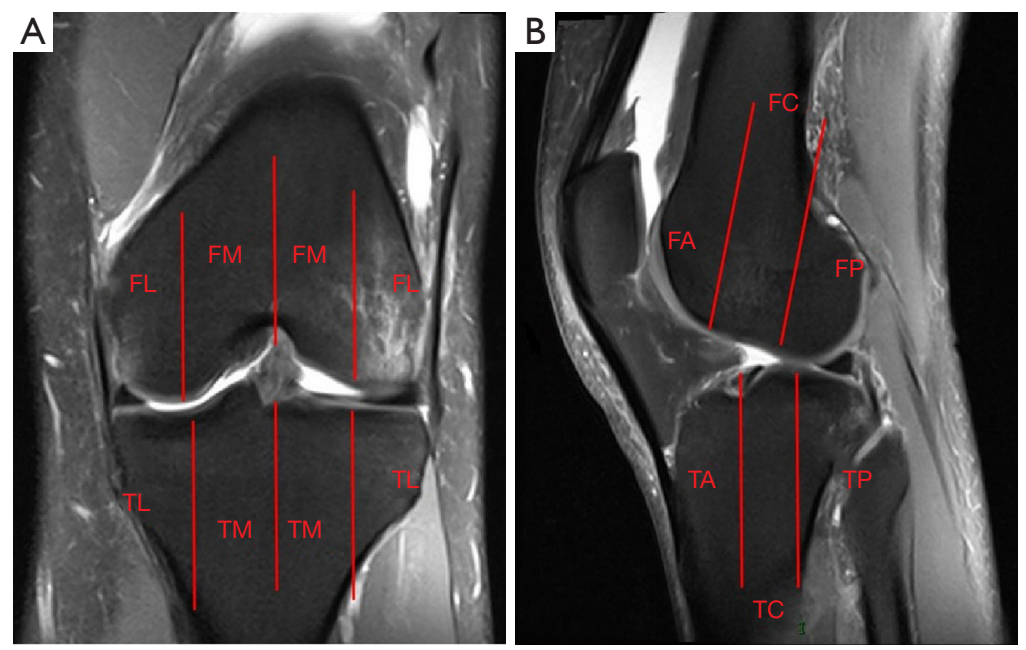

Figure 2 Bone contusion locations were determined in the femur and the tibia. (A) Bone contusion locations in the lateral-medial direction were determined using the coronal sequence. The lines were perpendicular to the subchondral bone of the femur and the tibia, and divided the four anatomical sites into two equal compartments, respectively. (B) Bone contusion locations in the anterior-posterior direction were determined using the sagittal sequence. The lines were perpendicular to the subchondral bone of the femur and the tibia, and divided it into three equal compartments. FM, femur-medial; FL, femur-lateral; TM, tibial plateau-medial; TL, tibial plateau-lateral; FA, femur-anterior; FC, femur-central; FP, femur-posterior; TA, tibial plateau-anterior; TC, tibial plateau-central; TP, tibial plateau-posterior.

Table 1 Classification of bone contusions on fat-suppressed magnetic resonance imagings

\begin{tabular}{ll}
\hline Grade & Definition \\
\hline Grade 1 (superficial) & Just beneath the subchondral bone \\
Grade 2 (shallow) & Extends up to one half of the distance from the articular surface to the physeal scar \\
Grade 3 (deep) & Extends up to two halfs of the distance from the articular surface to the physeal scar, but not beyond the scar \\
Grade 4 (generalized) & Extends beyond the physeal scar \\
\hline
\end{tabular}

reviewed by two musculoskeletal radiologists who were blinded to this study.

\section{Statistical analysis}

Statistical analyses were performed using SPSS software (version 25; IBM Corp., Armonk, NY, USA). Descriptive statistics, such as means, standard deviations, minimums, and maximums, were calculated for all continuous variables, including age. Fisher's exact test was used to compare the bone contusion distribution between the six subcompartments of each anatomic site. A kappa statistic was used to assess inter-observer agreement for magnetic resonance imaging studies on the depiction of bone contusions. A P value of $<0.05$ was considered statistically significant.

\section{Results}

\section{Descriptive statistics}

Ninety-three patients (aged 32.06 \pm 11.11 years) met the inclusion criteria and were enrolled in the study. The demographic factors of the patients are summarized in Table 2. The prevalence of bone contusions (as observed in magnetic resonance imaging scans) was as follows, $78.49 \%$ on the lateral femoral condyle, $88.17 \%$ on the lateral tibial plateau, $49.46 \%$ on the medial femoral condyle, and $69.89 \%$ on the medial tibial plateau. The distribution of bone contusions at four anatomical sites is shown in Figure 3. 
Table 2 Demographic factors of the patients

\begin{tabular}{lc}
\hline Characteristics & $\mathrm{n} / \mathrm{N}(\%)$ \\
\hline Age, years & $6 / 93(6.45)$ \\
$\leq 17$ & $87 / 93(93.55)$ \\
$\geq 18$ & \\
Gender & $64 / 93(68.82)$ \\
Male & $29 / 93(31.18)$ \\
Female & \\
Anatomical sites of bone contusions & $73 / 93(78.49)$ \\
Lateral femoral condyle & $82 / 93(88.17)$ \\
Lateral tibial plateau & $46 / 93(49.46)$ \\
Medial femoral condyle & $65 / 93(69.89)$ \\
Medial tibial plateau & \\
Kissing contusions & \\
Only the lateral side & $33 / 93(35.48)$ \\
Only the medial side & $5 / 93(5.38)$ \\
Both the lateral and medial sides & $34 / 93(36.56)$ \\
\hline
\end{tabular}

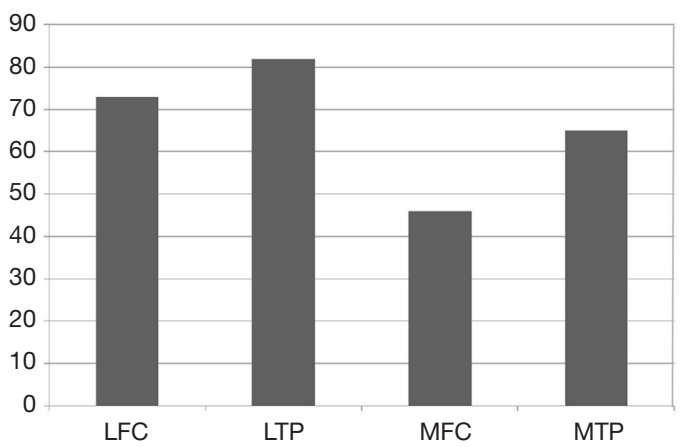

Figure 3 The distribution of bone contusions of each anatomical site in the knee. LFC, lateral femoral condyle; LTP, lateral tibial plateau; MFC, medial femoral condyle; MTP, medial tibial plateau.
Overall, 33 (35.48\%) knees had only lateral kissing contusions (LKCs), 5 (5.38\%) knees had only medial kissing contusions (MKCs), and 34 (36.56\%) knees had both LKCs and MKCs. The proportion of kissing contusions in the lateral compartment was higher than in the medial compartment (67 vs. 39, 72.04\% vs. 41.94\%). Kappa analyses of the magnetic resonance imaging determinations between the two blinded observers are shown in Table 3.

\section{Bone contusion patterns}

In this study, there was a significant difference in the subcompartments of the lateral tibial plateau in patients with LKCs $(\mathrm{P}=0.001)$. In these patients, bone contusions of the lateral tibial plateau occurred in a total of 76 subcompartments and were mainly located in the posterior aspect $(\mathrm{PM}, \mathrm{n}=22,28.95 \% ; \mathrm{PL}, \mathrm{n}=2,2.63 \% ; \mathrm{PL}+\mathrm{PM}$, $\mathrm{n}=43,56.57 \%)$. No significant differences were detected between the sub-compartments of the lateral femoral condyle in patients with LKCs; however, the distribution of bone contusions in compartments had a clear tendency. Of the 87 sub-compartments of the lateral femoral condyle, the most common location of bone contusions was the CL $(n=67$, $77.01 \%)$. Other common bone contusion locations were the $\mathrm{CM}(\mathrm{n}=11,12.64 \%)$, the $\mathrm{AL}(\mathrm{n}=5,5.75 \%)$, the PL $(\mathrm{n}=2$, $2.30 \%)$, the $\mathrm{PM}(\mathrm{n}=1,1.15 \%)$, and the $\mathrm{AM}(\mathrm{n}=1,1.15 \%)$ (see Figure $4 A$ ). In patients with MKCs, there were no significant differences between the sub-compartments. Bone contusions were observed in 41 sub-compartments at the medial femoral condyle and 42 sub-compartments at the medial tibial plateau. The most common locations of bone contusions on the medial femoral condyle was in the CL aspect $(\mathrm{n}=38,92.68 \%)$, and on the medial tibial plateau in the posterior aspect (PM, $\mathrm{n}=5,11.90 \%$; PL, $\mathrm{n}=19,45.25 \%$; PL + PM, $\mathrm{n}=14,33.33 \%$ ) (see Figure 4B) Additionally, anteromedial tibial contusions were found in two patients who had both MKCs and LKCs.

Table 3 Kappa analysis of magnetic resonance imaging determinations for bone contusions

\begin{tabular}{lcccc}
\hline \multirow{2}{*}{ Relevant factor } & \multicolumn{4}{c}{ Anatomical sites } \\
\cline { 2 - 4 } & Lateral femoral condyle & Llateral tibial plateau & Medial femoral condyl & Medial tibial plateau \\
\hline Kappa value & 0.693 & 0.752 & 0.806 & 0.800 \\
\hline
\end{tabular}


A

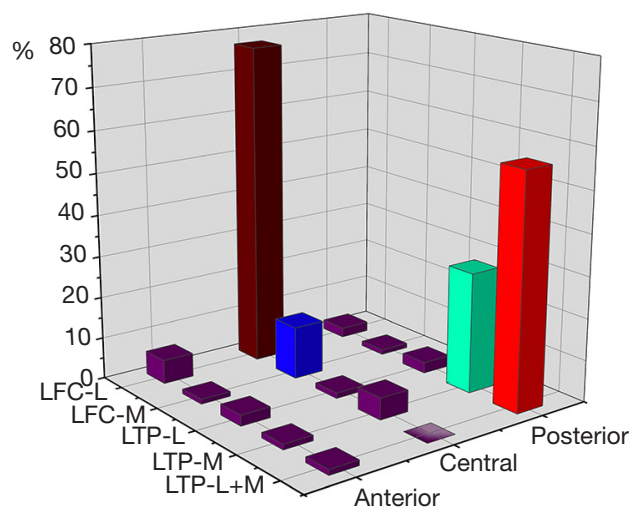

B

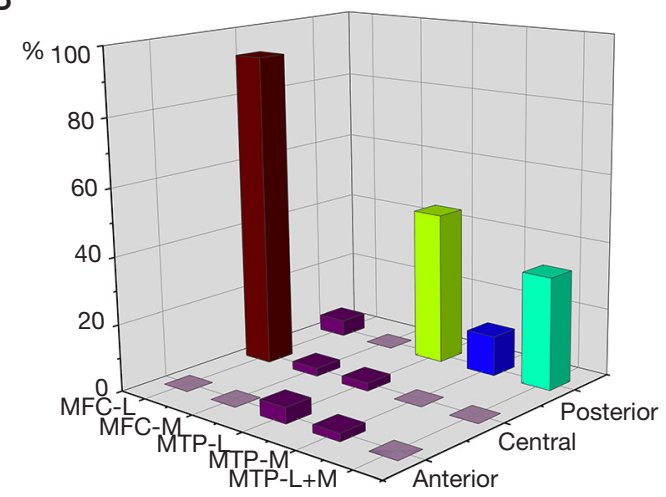

Figure 4 The prevalence of bone contusions for the four anatomical sites at the sub-compartments in patients with kissing contusions. (A) The prevalence of bone contusions of the LFC and the LTP at sub-compartments in patients with LKCs. (B) The prevalence of bone contusions of the MFC and the MTP at sub-compartments in patients with MKCs. LFC, lateral femoral condyle; LTP, lateral tibial plateau; LKC, lateral kissing contusion; MFC, medial femoral condyle; MTP, medial tibial plateau; MKC, medial kissing contusion.

A

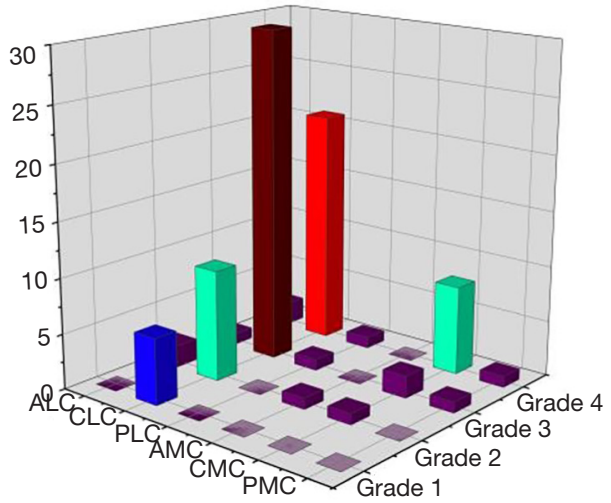

B

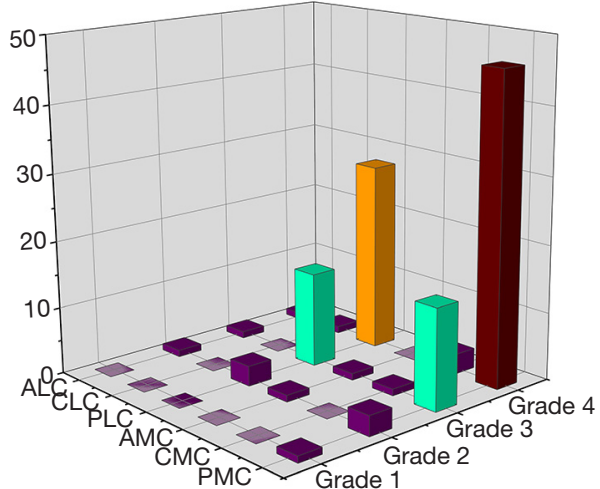

Figure 5 The severity of bone contusions at each sub-compartment in patients with LKCs. The severity of bone contusions at the six sub-compartments on the lateral femoral condyle (A) and lateral tibial plateau (B). LKC, lateral kissing contusion; ALC, anterior-lateral compartment; CLC, central-lateral compartment; PLC, posterior-lateral compartment; AMC, anterior-medial compartment; CMC, centralmedial compartment; PMC, posterior-medial compartment.

\section{Bone contusion severity}

The severity of bone contusions at each sub-compartment in patients with LKCs and MKCs are shown in Figures 5,6, respectively. In patients with LKCs, the most severe bone contusions on the lateral femoral condyle were all in the CL and the most severe bone contusions on the lateral tibial plateau were $88.06 \%$ (59 of 67 ) in the PM, and $11.94 \%$ (8 of 67) in the PL. For patients with MKCs, concerning the most severe bone contusions on the medial femoral condyle, $97.44 \%$ (38 of 39 ) were in the CL, and $2.56 \%$ ( 1 of 39 ) were in the CM. Concerning most severe bone contusions on the medial tibial plateau, $74.36 \%$ (29 of 39 ) were in the PL, $23.08 \%$ (9 of 39 ) in the PM, and $2.56 \%$ ( 1 of 39 ) were in the AM. Higher-grade bone contusions were more prevalent among patients with kissing contusions.

\section{Discussion}

In this study, we found that the primary mechanisms leading to non-contact anterior cruciate ligament injury based on the location patterns and severity of bone contusions were 
A

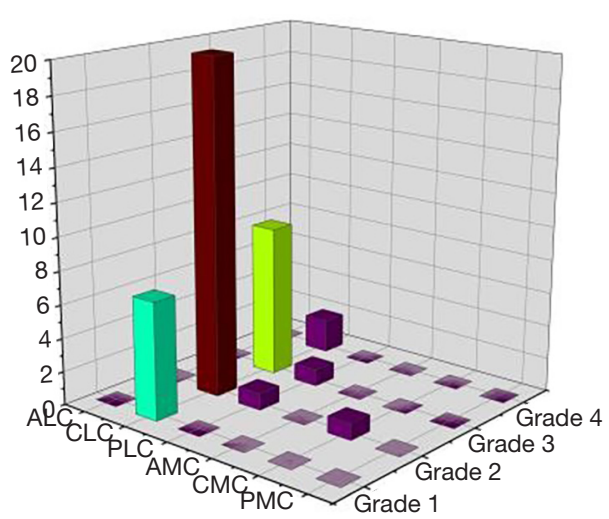

B

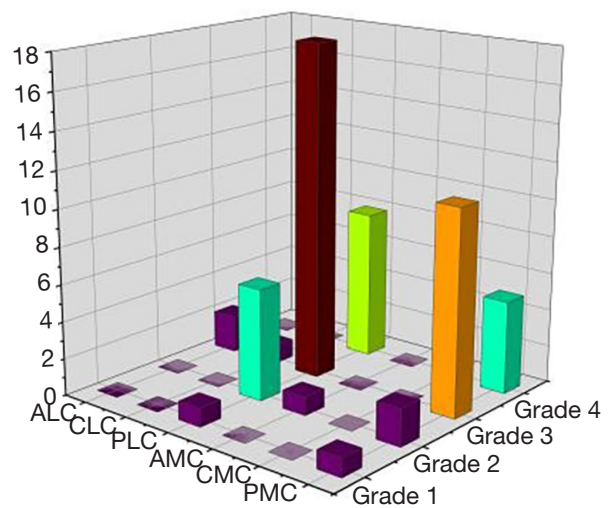

Figure 6 The severity of bone contusions at each sub-compartment in patients with MKCs. The severity of bone contusions at six subcompartments on the medial femoral condyle (A) and medial tibial plateau (B). MKC, medial kissing contusion; ALC, anterior-lateral compartment; CLC, central-lateral compartment; PLC, posterior-lateral compartment; AMC, anterior-medial compartment; CMC, centralmedial compartment; PMC, posterior-medial compartment.

A

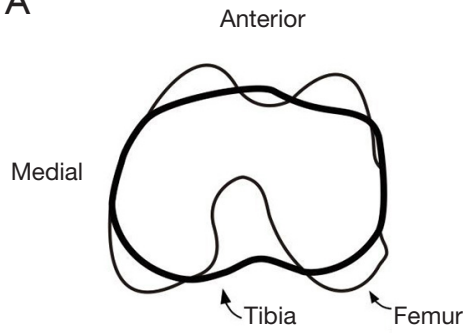

C

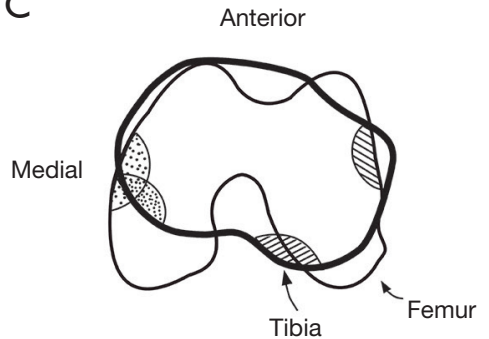

B

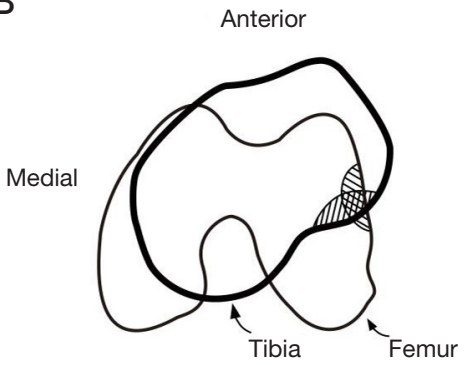

Anterior

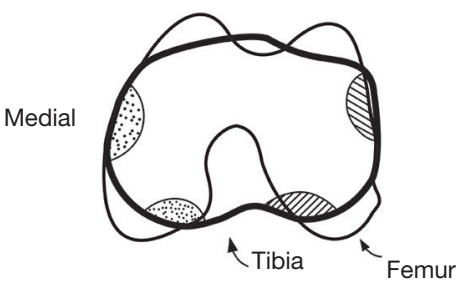

Figure 7 A diagram of non-contact anterior cruciate ligament injury cascade and the developing bone contusions. (A) Before injury, the tibia and the femur were in the normal positions without contusions. (B) During the early stages of injury, a coupled motion of valgus + lateral translation + anterior translation of the tibia and internal tibial rotation occurred that created an impact on the lateral articular surface. At the time of impact, lateral contusions (lined areas) occurred. (C) During the later stages of injury, a compensatory external rotation of the tibia and a sustaining anterior translation of the tibia resulted in bone contusions on the medial side (dotted areas). (D) After the injury, the tibia and the femur returned to the normal relative positions. The specific locations of the lateral and medial contusions are shown.

the tibia's internal rotation and the valgus knee accompanied by an anterior and lateral translation of the tibia. A diagram of the cascade of anterior cruciate ligament injuries is shown in Figure 7. First, we found that the most common bone contusion pattern observed among the patients was the lateral tibial plateau, followed by the lateral femoral condyle, the medial tibial plateau, and the medial femoral condyle. Tibiofemoral bone contusions on the lateral side were more common in patients with non-contact anterior cruciate ligament injuries than tibiofemoral bone contusions 
on the medial side. Second, the most common distribution pattern for bone contusions on the lateral femoral condyle and the medial femoral condyle was the CL. The most common distribution patterns for bone contusions were the $\mathrm{PM}$ in the lateral tibial plateau and the PL in the medial tibial plateau. Third, the most serious compartments were the PM on the lateral tibial plateau and the PL on the medial tibial plateau These findings provide important information about the mechanisms of non-contact anterior cruciate ligament injury.

The severity and the location patterns of tibiofemoral bone contusions observed on the magnetic resonance imaging scans may result from joint impact at the time of anterior cruciate ligament injury and could provide important information about anterior cruciate ligament injury mechanisms $(6,17,20,21)$. Previous studies have investigated the mechanisms of anterior cruciate ligament injuries using patterns of kissing bone contusions. These studies referred to the relative bone contusion patterns in both the tibia and femur and provide valuable indications about anterior cruciate ligament injury mechanisms based on a comparison to other bone contusion patterns $(22,23)$. In this study, $36.56 \%$ (34 of 93) of patients had both LKCs and MKCs. Similar to previous findings (17), we found that patients with only LKCs were more common than those with only MKCs (33 vs. 5, 35.48\% vs. 5.38\%). The overall increased prevalence of bone contusions at the lateral compartments may reflect the presence of valgus force at the time of anterior cruciate ligament injury that the medial side may cause "opening". These findings were consistent with previous studies that showed that valgus load is the primary mechanism of anterior cruciate ligament injuries $(23,24)$.

In this study, the locations of bone contusions at four anatomic sites were respectively subdivided into six compartments based on the lateral-medial and anteriorposterior directions. We found that kissing contusions occurred more commonly in the posterior aspect of the tibial plateau and the femoral condyle's central aspect. Consistent with previous studies $(2,17)$, lateral femoral condyle's most common pattern in patients with LKCs was the centrallateral. Previous studies have reported that tibial bone contusions occur in the posterior section. Sanders et al. (20) defined five contusion patterns in the knee as footprints to explore anterior cruciate ligament injury mechanisms. Knee pivot-shift injury is a non-contact injury that results in the lateral femoral condyle's impaction against the poster-lateral tibial plateau (20). The degree of flexion in the knee at the time of injury determines the exact location of the bone contusion on the lateral femoral condyle.

In our study, 56.57\% (43 of 76) of lateral tibial plateau contusions occurred in PM + PL in patients with LKCs. Interestingly, compared to the $\mathrm{PL}$, the $\mathrm{PM}$ was more commonly observed at the lateral tibial plateau $(28.95 \%$ vs. $2.63 \%$ ). The stress levels for injuries were not obtained; however, it was assumed that stress levels increased in patients without bone contusions on the lateral tibial plateau, in patients with bone contusions only in the PM, and in patients with a bone contusion in both the PM and the PL. The bone contusion distribution pattern of the lateral tibial plateau indicated that the direct point of impact was in the PM. These results suggest that the CL section of the lateral femoral condyle impacted the PM section of the lateral tibial plateau. Lateral bone contusions are often attributed to valgus stress; however, the valgus may not be the only force responsible for anterior cruciate ligament injury. Given our results, which were similar to those of Meyer et al. (25), we hypothesized that at the early stages of injury, the valgus's motion, the lateral translation, the anterior translation of tibia, and the internal tibial rotation were responsible for anterior cruciate ligament injury. Internal rotation stresses are present in the knee; however, the medial side of the lateral tibial plateau typically rotated to the lateral side and impacted the lateral femoral condyle. The changes place stress on the lateral compartment and on the anterior cruciate ligament that results in anterior cruciate ligament rupture.

Also, the severity of bone contusions in the six subcompartments on the knee's lateral side differed. The most severe bone contusions on the lateral femoral condyle were all in the CL, and those on the lateral tibial plateau were all in the PM. Based on the assumption that the impact's direct site was the most severe, the severity of other compartments in each site was less. The primary relative pattern of the tibiofemoral bone contusion was the PM on the lateral tibial plateau and the CL on the lateral femoral condyle. These results suggest that at the time of noncontact anterior cruciate ligament injury, the direct sites of the impact were the CL of the lateral femoral condyle and the PM of the lateral tibial plateau, which further indicated that an internal rotation of the tibia occurred during anterior cruciate ligament injury.

As bone contusions were more serious on the lateral section, the valgus was considered the primary injury mechanism (6). Other studies have reported that an anterior translation of the tibia relative to the femur with a small knee flexion angle may be the primary mechanism of an 
anterior cruciate ligament injury based on the observed bone contusions on the posterior tibia and anterior femur $(2,17)$. Further, the effects of the lateral translation of the tibia on anterior cruciate ligament injury has gradually attracted attention $(24,25)$. Ueno et al. used a six-axis kinetics simulator to investigate anterior cruciate ligament injury mechanisms and demonstrated that lateral tibial force increased during impact (26). In our study, bone contusions on the posterior lateral tibial plateau were more medial, and the severity of the medial section was higher than the lateral section. If only valgus and anterior tibial translation relative to the femur occurred when the anterior cruciate ligament was injured, the most severe location of the bone contusion of the lateral tibial plateau might not be common on the medial side. These data suggested there may be a lateral tibial force at the time of anterior cruciate ligament rupture. The asymmetric positions in the anteriorposterior and lateral-medial directions between the lateral and medial sides indicated that tibial rotation in the axial plane occurred during anterior cruciate ligament injury (17). When the tibia rotated internally, the posterior part of the lateral tibial plateau rotated to the lateral side and impacted the lateral femoral condyle, which caused the specific patterns observed in our study.

Similarly, previous studies on bone contusion patterns found that extension plays a role in non-contact anterior cruciate ligament injury $(2,22,23)$. Additionally, bone contusion patterns may be important in the position of the injury. Extension was commonly accompanied by an increased anterior tibial translation, internal tibial rotation, and valgus. Given these data, the extension mechanism may only be important when increased tibia and valgus movements occur.

The injury mechanism determined from the location and severity of bone contusions on the medial side was consistent with the lateral side. Only 5.38\% (5 of 93) of patients with a non-contact injury in our study had only MKCs. These data were similar to previous results that revealed that the prevalence of bone contusions on the medial side of the knee was significantly lower than that on the lateral side (2). Our data indicated that a non-contact anterior cruciate ligament injury may have a sustained impact between the lateral femoral condyle and the lateral tibial plateau and may have sustained less or no impact between the medial femoral condyle and the medial tibial plateau. The impact in the lateral side of the knee was more severe. Bone contusions on the posterior site of the medial tibial plateau may result from contrecoup impaction, as demonstrated in the study by Kaplan et al. (27).
Kaplan et al. conjectured that contusions on the medial side were caused by compensatory varus alignment and the femur's internal rotation after the initial external femoral rotation (27). However, Viskontas and Kim found that medial compartment contusions in non-contact injuries were attributed to anterior tibial translation and internal tibial rotation due to the medial side's relative location $(6,23)$. In our data, bone contusions on the medial side were commonly located in the PL of the medial tibial plateau and the CL of the medial femoral condyle; thus, we believe that the rotation of the tibia (rather than the femur) is the main mechanism of anterior cruciate ligament injury. During the later stages of injury, a compensatory external rotation of the tibia and a sustaining anterior translation of the tibia occurred that resulted in medial bone contusions. Approximately 50.75\% (34 of 67) of LKCs were accompanied by MKCs. In these patterns, the medial side's most severe locations were the CL on the medial femoral condyle and the PL on the medial tibial plateau. These may have resulted from a compensatory external rotation of the tibia after the tibia's initial internal rotation.

MKC was considerably less severe than LKC. The bone contusion difference of the location distribution and the severity between the lateral and the medial side can be explained by the dissipation of the initial impact of the bone in the lateral side. Compared with unilateral bone contusions, the force required to cause bilateral bone contusions may be greater. Wissman et al. $(28,29)$ found an uncommon contusion pattern in the patellotibial join. The anteromedial tibial contusions were thought to result from impaction between the inferior patella and the anteromedial tibial rim. In our study, anteromedial tibial contusion was only found in two patients who had both medial and LKCs with a high severity grade; thus, we speculated that anteromedial tibial contusion occurred only with larger impact forces. In this study, 29.03\% (27 of 93) of patients did not have kissing contusions. The pattern of bone contusions in the four anatomical sites was similar to that of kissing contusions; however, the number and severity of bone contusion compartments were lower in patients without kissing contusions. We also suggest that kissing bone contusions occur when the tibia's relative compressive force and the femur are sufficiently large.

This study had some limitations. First, it retrospectively evaluated magnetic resonance imaging data sets, and the knee kinematics data at the time of injury were not obtained. Second, while the prevalence of non-contact anterior cruciate ligament was higher in knee injuries, the 
number of patients included in the current study was small. Since the magnetic resonance imaging examination with an injury time of more than 1 month cannot correctly assess the existence and severity of the bone contusion, patients who underwent magnetic resonance imaging examination 1 month after the injury were excluded. Third, the anterior cruciate ligament injury pattern was complex. Further biomechanical evidence needs to be gathered to support our finding that anterior cruciate ligament injury mechanisms involved multiple factors rather than a single factor. Finally, this study focused only on the bone contusion location patterns and the severity and mechanisms of injury; however, the effects of the femur's anatomical features, the tibia, and the surrounding soft tissue on anterior cruciate ligament rupture were not considered.

In conclusion, bone contusions commonly occur on the lateral side, especially on the lateral tibial plateau. The CL of the lateral femoral condyle and the PM of the lateral tibial plateau were the most common and the most severe. Internal tibial rotation, valgus, tibial anterior, and lateral translation were shown to be the primary mechanisms of non-contact anterior cruciate ligament injury based on bone contusion patterns and severity. This study provides a novel method for characterizing the locations of bone contusions in the lateral-medial direction, which could make an important contribution to non-contact anterior cruciate ligament injuries. Our future research will focus on the effects of the knee's anatomical features and the knee position at the time of anterior cruciate ligament injury to explore the mechanism of injury with more DOF.

\section{Acknowledgments}

Funding: This work was supported by the Science and Technology Commission Project of Chongqing (Grant No. cstc2018jscx-mszdX0042).

\section{Footnote}

Conflicts of Interest: All authors have completed the ICMJE uniform disclosure form (available at http://dx.doi. org/10.21037/qims-20-1212). The authors have no conflicts of interest to declare.

Ethical Statement: This study was approved by the Institutional Review Board of The First Affiliated Hospital of Chongqing Medical University (No. 2017-108), and individual consent for this retrospective analysis was waived.
Open Access Statement: This is an Open Access article distributed in accordance with the Creative Commons Attribution-NonCommercial-NoDerivs 4.0 International License (CC BY-NC-ND 4.0), which permits the noncommercial replication and distribution of the article with the strict proviso that no changes or edits are made and the original work is properly cited (including links to both the formal publication through the relevant DOI and the license). See: https://creativecommons.org/licenses/by-nc-nd/4.0/.

\section{References}

1. Li K, Li J, Zheng X, Marot V, Murgier J, Cavaignac E, Huang $W$. Increased lateral meniscal slope is associated with greater incidence of lateral bone contusions in noncontact ACL injury. Knee Surg Sports Traumatol Arthrosc 2020;28:2000-8.

2. Zhang L, Hacke JD, Garrett WE, Liu H, Yu B. Bone bruises associated with anterior cruciate ligament injury as indicators of injury mechanism: a systematic review. Sports Med 2019;49:453-62.

3. Wetters N, Weber AE, Wuerz TH, Schub DL, Mandelbaum BR. Mechanism of injury and risk factors for anterior cruciate ligament injury. Oper Techn Sport Med 2016;24:2-6.

4. Alentorn-Geli E, Myer GD, Silvers HJ, Samitier G, Romero D, Lazaro-Haro C, Cugat R. Prevention of non-contact anterior cruciate ligament injuries in soccer players. Part 1: Mechanisms of injury and underlying risk factors. Knee Surg Sports Traumatol Arthrosc 2009; 17:705-29.

5. Krosshaug T, Nakamae A, Boden BP, Engebretsen L, Smith G, Slauterbeck JR, Hewett TE, Bahr R. Mechanisms of anterior cruciate ligament injury in basketball - Video analysis of 39 cases. Am J Sports Med 2007;35:359-67.

6. Viskontas DG, Giuffre BM, Duggal N, Graham D, Parker D, Coolican M. Bone bruises associated with ACL rupture: correlation with injury mechanism. Am J Sports Med 2008;36:927-33.

7. Koga H, Nakamae A, Shima Y, Iwasa J, Myklebust G, Engebretsen L, Bahr R, Krosshaug T. Mechanisms for noncontact anterior cruciate ligament injuries: knee joint kinematics in 10 injury situations from female team handball and basketball. Am J Sports Med 2010;38:2218-25.

8. Filardo G, Andriolo L, Frattura GDL, Napoli F, Zaffagnini S, Candrian C. Bone bruise in anterior cruciate ligament rupture entails a more severe joint damage affecting joint degenerative progression. Knee Surg Sports 
Traumatol Arthrosc 2019;27:44-59.

9. Markolf KL, Burchfield DM, Shapiro MM, Shepard MF, Finerman GA, Slauterbeck JL. Combined knee loading states that generate high anterior cruciate ligament forces. J Orthop Res 1995;13:930-5.

10. Lee CH, Tan CF, Kim O, Suh KJ, Yao M, Chan WP, Wu JS. Osseous injury associated with ligamentous tear of the knee. Can Assoc Radiol J 2016;67:379-86.

11. Mandalia V, Fogg A, Chari R, Murray J, Beale A, Henson J. Bone bruising of the knee. Clin Radiol 2005;60:627-36.

12. Gentili A, Seeger LL, Yao L, Do HM. Anterior cruciate ligament tear: indirect signs at MR imaging. Radiology 1994;193:835-40.

13. Yoon KH, Yoo JH, Kim K. Bone contusion and associated meniscal and medial collateral ligament injury in patients with anterior cruciate ligament rupture. J Bone Joint Surg Am 2011;93:1510-8.

14. Bastos R, Andrade R, Vasta S, Pereira R, Papalia R, van der Merwe W, Rodeo S, Espregueira-Mendes J. Tibiofemoral bone bruise volume is not associated with meniscal injury and knee laxity in patients with anterior cruciate ligament rupture. Knee Surg Sports Traumatol Arthrosc 2019;27:3318-26.

15. Calvo-Gurry M, Hurley ET, Withers D, Vioreanu M, Moran R. Posterior tibial bone bruising associated with posterior-medial meniscal tear in patients with acute anterior cruciate ligament injury. Knee Surg Sports Traumatol Arthrosc 2019;27:3633-7.

16. Gong J, Pedoia V, Facchetti L, Link TM, Ma CB, Li X. Bone marrow edema-like lesions (BMELs) are associated with higher $\mathrm{T} 1 \rho$ and $\mathrm{T} 2$ values of cartilage in anterior cruciate ligament (ACL)-reconstructed knees: a longitudinal study. Quant Imaging Med Surg 2016;6:661-70.

17. Shi H, Ding L, Jiang Y, Zhang H, Ren S, Hu X, Liu Z, Huang H, Ao Y. Bone bruise distribution patterns after acute anterior cruciate ligament ruptures: implications for the injury mechanism. Orthop J Sports Med 2020;8:2325967120911162.

18. Terzidis IP, Christodoulou AG, Ploumis AL, Metsovitis SR, Koimtzis M, Givissis P. The appearance of kissing contusion in the acutely injured knee in the athletes. Br J Sports Med 2004;38:592-6.

19. Shimokochi Y, Shultz SJ. Mechanisms of noncontact anterior cruciate ligament injury. J Athl Train 2008;43:396-408.

20. Sanders TG, Medynski MA, Feller JF, Lawhorn KW. Bone contusion patterns of the knee at MR imaging: footprint of the mechanism of injury. Radiographics 2000;20 Spec
No:S135-51.

21. Patel SA, Hageman J, Quatman CE, Wordeman SC, Hewett TE. Prevalence and location of bone bruises associated with anterior cruciate ligament injury and implications for mechanism of injury: a systematic review. Sports Med 2014;44:281-93.

22. Owusu-Akyaw KA, Kim SY, Spritzer CE, Collins AT, Englander ZA, Utturkar GM, Garrett WE, DeFrate LE. Determination of the position of the knee at the time of an anterior cruciate ligament rupture for male versus female patients by an analysis of bone bruises. Am J Sports Med 2018;46:1559-65.

23. Kim SY, Spritzer CE, Utturkar GM, Toth AP, Garrett WE, DeFrate LE. Knee Kinematics During Noncontact Anterior Cruciate Ligament Injury as Determined From Bone Bruise Location. Am J Sports Med 2015;43:2515-21.

24. Mazzocca AD, Nissen CW, Geary M, Adams DJ. Valgus medial collateral ligament rupture causes concomitant loading and damage of the anterior cruciate ligament. J Knee Surg 2003;16:148-51.

25. Meyer EG, Haut RC. Anterior cruciate ligament injury induced by internal tibial torsion or tibiofemoral compression. J Biomech 2008;41:3377-83.

26. Ueno R, Navacchia A, Bates NA, Schilaty ND, Krych AJ, Hewett TE. Analysis of internal knee forces allows for the prediction of rupture events in a clinically relevant model of anterior cruciate ligament injuries. Orthop J Sports Med 2020;8:2325967119893758.

27. Kaplan PA, Gehl RH, Dussault RG, Anderson MW, Diduch DR. Bone contusions of the posterior lip of the medial tibial plateau (contrecoup injury) and associated internal derangements of the knee at MR imaging. Radiology $1999 ; 211: 747-53$.

28. Wissman RD, England E, Mehta K, Burch M, Javadi A, Newton K. The anteromedial tibial rim sign: an indicator of patellotibial impaction in acute anterior cruciate ligament tears. J Comput Assist Tomogr 2015;39:57-63.

29. Wissman RD, England E, Mehta K, Nepute J, Von Fischer $\mathrm{N}$, Apgar J, Javadi A. Patellotibial contusions in anterior cruciate ligament tears. Skeletal Radiol 2014;43:247-50.

Cite this article as: Qiu L, Sheng B, Li J, Xiao Z, Yuan M, Yang H, Lv F, Lv F. Mechanisms of non-contact anterior cruciate ligament injury as determined by bone contusion location and severity. Quant Imaging Med Surg 2021;11(7):32633273. doi: 10.21037/qims-20-1212 